\title{
PROVABILITY WITH FINITELY MANY VARIABLES
}

\section{J. DONALD MONK}

ABstract. In first-order logic with equality but with finitely many variables, no finite schema suffices to give a sound and complete axiomatization of the universally valid sentences. The proof uses a rather deep result from algebraic logic.

The purpose of this note is to give a rather obvious consequence of a recent theorem of J. S. Johnson [5] (whose proof is based on Monk [7]). The consequence has to do with provability in languages which differ from ordinary first-order languages with equality just in having only finitely many individual variables. Such languages have previously been investigated in Henkin [2], Henkin-Tarski [3], Jaśkowski [4], and Pieczkowski [8]. Our result is that it is impossible to write down finitely many schemata which will give a notion of proof that is sound and complete. See below for a more precise formulation; in particular, the notion of schema is made explicit.

What makes our result an easy consequence of Johnson's theorem is the fairly general knowledge of certain connections between logically valid sentences in these restricted first-order languages, and equations which hold identically in each representable polyadic equality algebra. The main portion of the note is devoted to an exposition of these connections. Thanks are due to the referee for aid in the formulation of these connections.

We employ the usual set-theoretic notation. $f^{*} X$ is the $f$-image of the set $X . \omega$ is the set of all natural numbers. We assume throughout that $3 \leqq \alpha<\omega$ (Our languages will have $\alpha$ variables; the case $\alpha \leqq 2$ is considered in Henkin [2].)

$\mathscr{L}^{\alpha}$ is the first-order language with equality with the sequence $\left\langle v_{i}: i\langle\alpha\rangle\right.$ of individual variables and with the sequence $\left\langle\boldsymbol{R}_{i}: i\langle\omega\rangle\right.$ of nonlogical constants, where $\boldsymbol{R}_{i}$ is an $\alpha$-ary relation symbol for each $i<\omega$. We treat $\neg, \rightarrow, \forall$, and $=$ as primitive logical symbols; $\mathbf{V}, \Lambda, \leftrightarrow$, and $\exists$ are defined in the usual way. An $\mathscr{L}^{\alpha}$-structure is a structure $\mathfrak{A}=\left\langle A, R_{i}\right\rangle_{i<\omega}$ where $A \neq 0$ and $R_{i} \subseteq{ }^{\alpha} A$ for each $i<\omega$. If

Received by the editors November 10. 1969 and, in revised form, March 13, 1970.

AMS 1969 subject classifications. Primary 0216, 0218; Secondary 0248.

Key words and phrases. Provability, first-order logic, polyadic algebras, set polyadic algebras, representable polyadic algebras, logically valid sentences, equations holding in polyadic algebras. 
$\phi$ is a formula of $\mathscr{L}^{\alpha}$ and $\mathfrak{A}$ is an $\mathscr{L}^{\alpha}$-structure, we denote by $\tilde{\phi}^{\mathfrak{Q}}$ the set of all sequences $x \in{ }^{\alpha} A$ which satisfy $\phi$ in $\mathfrak{A}$. If $\tilde{\phi}^{\mathfrak{A}}={ }^{\alpha} A$, we write $\mathfrak{A} \vDash \phi$, and if $\mathfrak{A} \vDash \phi$ for all $\mathfrak{A}$ we write $\vDash \phi ; \phi$ is then called universally valid. For our further purposes it is important to have a general notion of substitution in formulas. Let $\tau \in^{\alpha} \alpha$ and let $\phi$ be a formula of $\mathscr{L}^{\alpha}$. We wish to define $S(\tau) \phi$, the $\tau$-substitution of $\phi$. It is the result of substituting simultaneously $v_{r i}$ for $v_{i}$ for each $i<\alpha$, first renaming bound variables. Explicitly:

$$
\begin{aligned}
S(\tau) R_{j} v_{\sigma 0} \cdots v_{\sigma(\alpha-1)} & =R_{j} v_{\tau \sigma 0} \cdots v_{\tau \sigma(\alpha-1)} \text { for } j \in \omega \text { and } \sigma \in{ }^{\alpha} \alpha \\
S(\tau)\left(v_{i}=v_{j}\right) & =v_{\tau i}=v_{\tau j} ; \\
S(\tau)(\rceil \phi) & =\rceil \delta(\tau) \phi ; \\
S(\tau)(\phi \rightarrow \psi) & =S(\tau) \phi \rightarrow \delta(\tau) \psi ; \\
S(\tau) \forall v_{i} \phi & =\forall v_{j} \delta(\sigma) \phi,
\end{aligned}
$$

where $j$ is the least element of $\alpha \sim \tau^{*}(\alpha \sim\{i\}), \sigma|\alpha \sim\{i\}=\tau| \alpha \sim\{i\}$, and $\sigma i=j$. It is easily verified that $\mathrm{s}(\tau) \phi$ is a proper substitution:

Lemma 1. If $\mathfrak{A}$ is an $\mathfrak{L}^{\alpha}$-structure, $x \in{ }^{\alpha} A, \phi$ is a formula of $\mathfrak{L}^{\alpha}$, and $\tau \in{ }^{\alpha} \alpha$, then $x$ satisfies $\mathcal{S}(\tau) \phi$ in $\mathfrak{A}$ iff $x \circ \tau$ satisfies $\phi$ in $\mathfrak{A}$.

We also need the notion of an instance of a formula of $\mathfrak{L}^{\alpha}$. Let $\phi$ be a function mapping $\omega$ into the set of formulas of $\mathscr{L}^{\alpha}$. For any formula $\psi$ of $\mathscr{L}^{\alpha}$ we define a formula $\delta(\phi, \psi)$ called the $\phi$-instance of $\psi$ in $\mathscr{L}^{\alpha}$ :

$$
\begin{aligned}
\delta\left(\phi, R_{i} v_{\sigma 0} \cdots v_{\sigma(\alpha-1)}\right) & =\delta(\sigma) \phi_{i} \text { for } i<\omega ; \\
\delta\left(\phi, v_{i}=v_{j}\right) & =v_{i}=v_{j} ; \\
\delta(\phi, \neg \psi) & =\rceil \delta(\phi, \psi) ; \\
\delta(\phi, \psi \rightarrow \chi) & =\delta(\phi, \psi) \rightarrow \delta(\phi, \chi) ; \\
\delta\left(\phi, \forall v_{i} \psi\right) & =\forall v_{i} \delta(\phi, \psi) .
\end{aligned}
$$

If $\Gamma$ is a set of formulas of $\mathscr{L}^{\alpha}$, we let $\operatorname{Inst}(\Gamma)$ be the set of all instances of formulas of $\Gamma$ in $\mathfrak{L}^{\alpha}$. We say that $\mathfrak{L}^{\alpha}$ is axiomatizable by a schema $\Gamma$ provided that the closure of $\operatorname{Inst}(\Gamma)$ under detachment and generalization consists exactly of all universally valid formulas of $\mathfrak{L}^{\alpha}$. Now we can state our main result:

THEOREM. There is no finite schema which axiomatizes $\mathfrak{L}^{\alpha}$.

As we mentioned at the outset, the proof of the theorem depends on some connections between $\mathfrak{L}^{\alpha}$ and a language for polyadic equality 
algebras. A polyadic equality algebra of dimension $\alpha\left(\mathrm{a} \mathbf{P E A}_{\alpha}\right)$ is a structure

$$
\mathfrak{A}=\left\langle A,+, \cdot,-, 0,1, S(\tau), \exists(\Gamma), d_{i j}\right\rangle_{\tau \in \alpha_{\alpha}, \Gamma \subseteq \alpha, i<\alpha, j<\alpha}
$$

satisfying the usual axioms (cf. Halmos [1]). A set polyadic equality algebra of dimension $\alpha$ (an SPEA $\mathbf{A}_{\alpha}$ ) is a $\mathbf{P E A}_{\alpha} \mathfrak{A}$ such that for some set $U$ (called the domain of $\mathfrak{X}$ ),

$$
\mathfrak{A}=\left\langle A, \cup, \cap, \sim, 0,{ }^{\alpha} U, S(\tau), \exists(\Gamma), d_{i j}\right\rangle_{\tau \in \alpha_{\alpha}, \Gamma \subseteq \alpha, i}<_{\alpha, j}<_{\alpha}
$$

where

$$
\begin{aligned}
& S(\tau) X=\left\{u \in{ }^{\alpha} U: u \circ \tau \in X\right\} ; \\
& \exists(\Gamma) X=\left\{u \in{ }^{\alpha} U: \text { there is a } v \in X\right. \text { such that } \\
& \qquad u \uparrow \alpha \sim \Gamma=v \uparrow \alpha \sim \Gamma\} ; \\
& d_{i j}=\left\{u \in{ }^{\alpha} U: u_{i}=u_{j}\right\} .
\end{aligned}
$$

A PEA $\mathbf{A}_{\alpha} \mathfrak{U}$ is representable ( $\mathfrak{U} \in \mathbf{R P E A _ { \alpha }}$ ) if $\mathfrak{U}$ is isomorphic to a subdirect product of SPEA $_{\alpha}$ 's. Note that our notion of a set polyadic equality algebra of dimension $\alpha$ is essentially the same as Halmos' notion of an 0 -valued functional $\alpha$-algebra with functional equality. A simple $\mathbf{P E A _ { \alpha }}$ is representable iff it is isomorphic to an $\mathbf{S P E A _ { \alpha }}$. We let $\rho^{\alpha}$ be an ordinary first-order language with equality suitable for PEA $A_{\alpha}$ 's, say with the sequence $\left\langle w_{i}: i\langle\omega\rangle\right.$ of individual variables. If $\sigma$ is a term of $\rho^{\alpha}, \mathfrak{A}$ is a $\operatorname{PEA}_{\alpha}$, and $x \in \in^{\omega} A$, we denote by $\tilde{\sigma}^{\mathfrak{x}} x$ the value of $\sigma$ in $\mathfrak{A}$ under the assignment of the value $x_{i}$ to $v_{i}$ for each $i<\omega$. An equation $\sigma=\tau$ holds in a PEA $\mathfrak{A}$ provided that $\tilde{\sigma}^{\mathfrak{A}} x=\tilde{\tau}^{\mathfrak{A}} x$ for all $x \in{ }^{\omega} A ; \mathfrak{A}$ is then called a model of $\sigma=\tau$. If $\Gamma$ is a set of equations, then $\mathfrak{A}$ is a model of $\Gamma$ if it is a model of each member of $\Gamma$.

Now we can set up some connections between $\mathcal{L}^{\alpha}$ and $\boldsymbol{p}^{\alpha}$. We define a mapping $T$ from formulas of $\mathfrak{S}^{\alpha}$ into terms of $\rho^{\alpha}$ :

$$
\begin{aligned}
T R_{i} v_{\tau 0} \cdots v_{\tau(\alpha-1)} & =S(\tau) w_{i} \text { for } i<\omega ; \\
T\left(v_{i}=v_{j}\right) & =d_{i j} ; \\
T(\rceil \phi) & =-T \phi ; \\
T(\phi \rightarrow \psi) & =-T \phi+T \psi ; \\
T \forall v_{i} \phi & =-\exists(\{i\})-T \phi .
\end{aligned}
$$

Going the other way we define a mapping $\Phi$ from terms of $\rho^{\alpha}$ to formulas of $\mathfrak{L}^{\alpha}$ : 


$$
\begin{aligned}
\Phi w_{i} & =R_{i} v_{0} \cdots v_{\alpha-1} \\
\Phi(-\sigma) & =\rceil \Phi \sigma ; \\
\Phi(\sigma+\tau) & =\Phi \sigma \vee \Phi \tau ; \\
\Phi(\sigma \cdot \tau) & =\Phi \sigma \wedge \Phi \psi ; \\
\Phi 0 & =\rceil v_{0}=v_{0} ; \\
\Phi 1 & =v_{0}=v_{0} ; \\
\Phi S(\tau) \sigma & =S(\tau) \Phi \sigma ; \\
\Phi \exists(\Gamma) \sigma & =\exists v_{\sigma 0} \cdots \exists v_{\sigma(m-1)} \Phi \sigma
\end{aligned}
$$

where $\Gamma=\{\sigma 0, \cdots, \sigma(m-1)\}$ with $\sigma 0<\cdots<\sigma(m-1)$;

$$
\Phi d_{i j}=v_{i}=v_{j}
$$

If $\mathfrak{A}$ is an $\mathscr{L}^{\alpha}$-structure, then $\left\{\tilde{\phi}^{\mathfrak{A}}: \phi\right.$ a formula of $\left.\mathscr{L}^{\alpha}\right\}$ is clearly the universe of an SPEA $\mathbf{A}_{\alpha}$, which will be denoted by $\mathfrak{A}$. By induction on $\phi$ one shows:

LEMMA 2. If $\mathfrak{A}=\left\langle A, R_{i}\right\rangle_{i<\omega}$ is an $\mathfrak{L}^{\alpha}$-structure and $\phi$ is an $\mathscr{L}^{\alpha}$-formula, then

$$
\tilde{\phi}^{\mathfrak{A}}=\widetilde{\boldsymbol{\phi}^{\tilde{\mathfrak{A}}}} R \text {. }
$$

If $\mathscr{B}$ is an SPEA $A_{\alpha}$ with domain $A$ and $R \in{ }^{\omega} B$, we set $\bar{B}^{R}$ $=\left\langle A, R_{i}\right\rangle_{i<\omega}$. Thus $\overline{\mathfrak{B}}^{R}$ is an $\mathscr{L}^{\alpha}$-structure. Again using induction on $\phi$ we have:

Lemma 3. If $\mathfrak{B}$ is an $\operatorname{SPEA}_{\alpha}, R \in{ }^{\omega} B$, and $\phi$ is an $\mathfrak{L}^{\alpha}$-formula, then

$$
\tilde{\phi}^{\overline{\mathfrak{P}}^{R}}=\widetilde{T} \phi^{\mathfrak{B}} R \text {. }
$$

Actually Lemma 3 can easily be derived from Lemma 2 since $\overline{\mathfrak{B}}^{R}$ is clearly a subalgebra of $\mathfrak{B}$. From Lemmas 2 and 3 we easily obtain:

Lemma 4. For any formula $\phi$ of $\mathfrak{L}^{\alpha}$ the following two conditions are equivalent:

(i) $\vDash \phi$;

(ii) $T \phi=1$ holds in every RPEA$_{\alpha}$.

Lemma 5. For any formulas $\phi, \psi$ of $\mathscr{L}^{\alpha}$ the following two conditions are equivalent:

(i) $\vDash \phi \leftrightarrow \psi$;

(ii) $T \phi=T \psi$ holds in every $\mathbf{R P E A}_{\boldsymbol{\alpha}}$.

The following lemmas are established analogously: 
LeMmA 6. If $\sigma$ is a term of $\mathcal{P}^{\alpha}, \mathfrak{B}$ is an $\mathrm{SPEA}_{\alpha}$, and $R \in^{\omega} B$, then

$$
\tilde{\sigma} \mathfrak{B} R=\widetilde{\Phi} \bar{\sigma}^{\mathbb{B}} \text {. }
$$

Lемма 7. If $\sigma$ is a term of $\mathcal{P}^{\alpha}$ and $\mathfrak{U}=\left\langle A, R_{i}\right\rangle_{i<\omega}$ is an $\mathfrak{L}^{\alpha}$-structure, then

$$
\tilde{\sigma^{\tilde{\mathfrak{A}}} R}=\widetilde{\Phi} \tilde{\sigma}^{\mathscr{A}} .
$$

LEMMA 8. For any term $\sigma$ of $p^{\alpha}$ the following two conditions are equivalent:

(i) $\sigma=1$ holds in every RPEA ;

(ii) $\vDash \Phi \sigma$.

LEMMA 9. For any terms $\sigma, \tau$ of $\rho^{\alpha}$ the following two conditions are equivalent:

(i) $\sigma=\tau$ holds in every RPEA ;

(ii) $\vDash \Phi \sigma \leftrightarrow \Phi_{\tau}$.

By an easy induction on $\phi$ we obtain the following two lemmas:

LEMMA 10. If $\phi$ is any formula of $\mathfrak{L}^{\alpha}$, then $\vDash \phi \leftrightarrow \Phi T \phi$.

LEMMA 11. If $\phi$ is any formula of $\mathscr{L}^{\alpha}$ and $\tau \in^{\alpha} \alpha$, then $T S(\tau) \phi=S(\tau) T \phi$ holds in any $\mathbf{P E A}_{\alpha}$.

By an easy induction on $\sigma$ using Lemma 11 we get:

LEMMA 12. If $\sigma$ is any term of $\rho^{\alpha}$, then $\sigma=T \Phi \sigma$ holds in every $\mathbf{P E A}_{\alpha}$.

Also, induction on $\psi$ using Lemma 11 yields:

LEMMA 13. If $\phi$ maps $\omega$ into the set of formulas of $\mathscr{L}^{\alpha}, \psi$ is a formula of $\mathscr{L}^{\alpha}, \mathfrak{B}$ is a $\mathrm{PEA}_{\alpha}$, and $R \in{ }^{\omega} B$, then

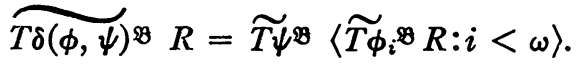

Finally, the following lemma obviously has our theorem as a corollary, using Theorem 3.5 of Johnson [5].

LEMMA 14. If $\mathfrak{L}^{\alpha}$ is axiomatizable by a schema $\Gamma$, then $\mathbf{R P E A}_{\alpha}$ is the set of all models of $\Gamma^{\prime}$, where $\Gamma^{\prime}=\{T \phi=1: \phi \in \Gamma\}$.

Proof. If $\phi \in \Gamma$, then $\vDash \phi$, and hence $T \phi=1$ holds in every RPEA $A_{\alpha}$,

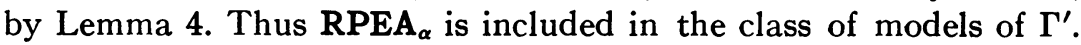
Conversely, suppose that $\mathfrak{B}$ is a model of $\Gamma^{\prime}$. To show that $\mathfrak{B} \in \mathbf{R P E A}_{\alpha}$, we shall make use of the fact, essentially due to Tarski (cf. Tarski [9]), that $\mathbf{R P E A}_{\alpha}$ is an equational class. In fact, $\mathbf{R P E A _ { \alpha }}$ can be characterized by a set of equations of the form $\sigma=1$. Let, then, $\sigma=1$ be an 


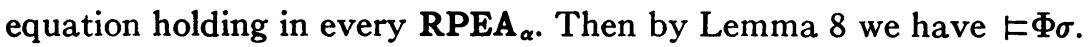
Now

(1) for any formula $\psi$ of $\mathfrak{L}^{\alpha}$, if $\vDash \psi$, then $T \psi=1$ holds in $\mathfrak{B}$.

In fact, let $\Delta=\{\psi: T \psi=1$ holds in $\mathscr{B}\}$. If $\phi$ maps $\omega$ into the set of formulas of $\mathscr{L}^{\alpha}$ and $\psi \in \Gamma$, we easily infer from Lemma 13 and the fact that $B$ is a model of $\Gamma^{\prime}$ that $\delta(\phi, \psi) \in \Delta$. Hence $\operatorname{Inst}(\Gamma) \subseteq \Delta$. Since $\Delta$ is clearly closed under detachment and generalization, it follows from the hypothesis of the lemma that $\{\phi: \vDash \phi\} \subseteq \Delta$. Hence (1) holds.

By (1), $T \Phi \sigma=1$ holds in $\mathfrak{B}$. From Lemma 12 the desired resultthat $\sigma=1$ holds in $\mathfrak{B}$-now follows.

\section{REFERENCES}

1. P. R. Halmos, Algebraic logic, Chelsea, New York, 1962. MR 24 \#A1808.

2. L. Henkin, Logical systems containing only a finite number of symbols, Séminaire Mathématiques Supérieures, no. 21, Presses Univ. Montreal, Montreal, 1967. MR 39 \#1313.

3. L. Henkin and A. Tarski, Cylindric algebras, Proc. Sympos. Pure Math., vol. 2, Amer. Math. Soc., Providence, R.I., 1961, pp. 83-113. MR 23 \#A1564.

4. S. Jaskowski, Sur les variables propositionnelles dépendantes, Studia Soc. Sci. Torunensis Sect. A. 1 (1948), 17-21. MR 10, 2.

5. J. S. Johnson, Nonfinitizability of classes of representable polyadic algebras, J. Symbolic Logic 34 (1969), 344-352.

6. D. Kalish and R. Montague, On Tarski's formalization of predicate logic with identity, Arch. Math. Logik Grundlagenforsch. 7 (1965), 81-101, MR 34 \#2438.

7. J. D. Monk, Nonfinitizability of classes of representable cylindric algebras, J. Symbolic Logic 34 (1969), 331-343.

8. A. Pieczkowski, Undecidability of the homogeneous formulas of degree 3 of the predicate calculus, Studia Logica 22 (1968), 7-16. MR 38 \#4311.

9. A. Tarski, Contributions to the theory of models. III, Nederl. Akad. Wetensch. Proc. Ser. A 57 = Indag. Math. 17 (1955), 56-64. MR 16, 554.

University of Colorado, Boulder, Colorado 80302 